\title{
Some New Families of Prime Cordial Graphs
}

\author{
S K Vaidya (Corresponding author) \\ Department of Mathematics, Saurashtra University \\ Rajkot 360005, Gujarat, India \\ E-mail: samirkvaidya@yahoo.co.in \\ N H Shah \\ V.V.P. Engineering College \\ Rajkot 360005, Gujarat, India \\ E-mail: nirav.hs@gmail.com
}

Received: March 1, 2011 Accepted: March 18, 2011 Published: November 1, 2011

doi:10.5539/jmr.v3n4p21 URL: http://dx.doi.org/10.5539/jmr.v3n4p21

\begin{abstract}
In this paper some new families of prime cordial graphs are investigated. We prove that the square graph of path $P_{n}$ is a prime cordial graph for $n=6$ and $n \geq 8$ while the square graph of cycle $C_{n}$ is a prime cordial graph for $n \geq 10$. We also show that the shadow graph of $K_{1, n}$ for $n \geq 4$ and the shadow graph of $B_{n, n}$ are prime cordial graphs. Moreover we prove that the graphs obtained by mutual duplication of a pair of edges as well as mutual duplication of a pair of vertices from each of two copies of cycle $C_{n}$ admit prime cordial labeling.
\end{abstract}

Keywords: Prime cordial labeling, Square Graph, Shadow Graph

AMS Subject classification number(2010): 05C78.

\section{Introduction}

We begin with simple, finite, connected and undirected graph $G=(V(G), E(G))$ with $p$ vertices and $q$ edges. For standard terminology and notations we follow (Gross, J., Yellen, J. , 1999). We will provide brief summary of definitions and other information which are necessary for the present investigations.

1.1 Definition If the vertices are assigned values subject to certain condition(s) then it is known as graph labeling.

For a dynamic survey on various graph labeling problems along with extensive bibliography we refer to (Gallian J. A., 2010).

1.2 Definition A mapping $f: V(G) \rightarrow\{0,1\}$ is called binary vertex labeling of $G$ and $f(v)$ is called the label of the vertex $v$ of $G$ under $f$.

1.3 Notations If for an edge $e=u v$, the induced edge labeling $f^{*}: E(G) \rightarrow\{0,1\}$ is given by $f^{*}(e)=|f(u)-f(v)|$. Then

$$
\left.\begin{array}{ll}
v_{f}(i)= & \text { number of vertices of } G \text { having label } i \text { under } f \\
e_{f}(i)= & \text { number of edges of } G \text { having label } i \text { under } f^{*}
\end{array}\right\} \text { where } i=0 \text { or } 1
$$

1.4 Definition A binary vertex labeling $f$ of a graph $G$ is called a cordial labeling if $\left|v_{f}(0)-v_{f}(1)\right| \leq 1$ and $\left|e_{f}(0)-e_{f}(1)\right| \leq 1$. A graph $G$ is cordial if it admits cordial labeling.

The concept of cordial labeling was introduced in (Cahit, I., 1987, p.201-207). After this many researchers have investigated graph families or graphs which admit cordial labeling. Some labeling schemes are also introduced with minor variations in cordial theme. Product cordial labeling, total product cordial labeling and prime cordial labeling are among mention a few. The present work is focused on prime cordial labeling.

1.5 Definition A prime cordial labeling of a graph $G$ with vertex set $V(G)$ is a bijection $f: V(G) \rightarrow\{1,2,3, \ldots,|V(G)|\}$ and the induced function $f^{*}: E(G) \rightarrow\{0,1\}$ is defined by

$$
\begin{aligned}
f^{*}(e=u v) & =1 ; & & \text { if } \operatorname{gcd}(f(u), f(v))=1 \\
& =0 ; & & \text { otherwise }
\end{aligned}
$$

satisfies the condition $\left|e_{f}(0)-e_{f}(1)\right| \leq 1$. A graph which admits prime cordial labeling is called a prime cordial graph. 
The concept of prime cordial labeling was introduced in (Sundaram, M., Ponraj, R. and Somasundram, S., 2005, p.373390) and they have investigated several results on prime cordial labeling. Prime cordial labeling in the context of graph operations is discussed in (Vaidya, S. K. and Vihol, P. L., 2010, p.119-126). The same authors have discussed prime cordial labeling for some cycle related graphs in (Vaidya, S.K. and P.L.Vihol, 2010, p. 223-232). In the present work we will investigate some new families of prime cordial graphs.

1.6 Definition For a simple connected graph $G$ the square of graph $G$ is denoted by $G^{2}$ and defined as the graph with the same vertex set as of $G$ and two vertices are adjacent in $G^{2}$ if they are at a distance 1 or 2 apart in $G$.

1.7 Definition The shadow graph $D_{2}(G)$ of a connected graph $G$ is obtained by taking two copies of $G$ as $G^{\prime}$ and $G^{\prime \prime}$. Join each vertex $u^{\prime}$ in $G^{\prime}$ to the neighbours of the corresponding vertex $u^{\prime \prime}$ in $G^{\prime \prime}$.

1.8 Definition Bistar is the graph obtained by joining the apex vertices of two copies of star $K_{1, n}$.

1.9 Definition Consider two copies of cycle $C_{n}$. Then the mutual duplication of a pair of vertices $v_{k}$ and $v_{k}^{\prime}$ from each of two copies of cycle $C_{n}$ produces a new graph $G$ such that $N\left(v_{k}\right)=N\left(v_{k}^{\prime}\right)$.

1.10 Definition Consider two copies of cycle $C_{n}$ and let $e_{k}=v_{k} v_{k+1}$ be an edge in the first copy of $C_{n}$ with $e_{k-1}=v_{k-1} v_{k}$ and $e_{k+1}=v_{k+1} v_{k+2}$ be its incident edges. Similarly let $e_{k}^{\prime}=u_{k} u_{k+1}$ be an edge in the second copy of $C_{n}$ with $e_{k-1}^{\prime}=u_{k-1} u_{k}$ and $e_{k+1}^{\prime}=u_{k+1} u_{k+2}$ be its incident edges. The mutual duplication of a pair of edges $e_{k}$ and $e_{k}^{\prime}$ from each of two copies of cycle $C_{n}$ produces a new graph $G$ in such a way that $N\left(v_{k}\right) \cap N\left(u_{k}\right)=\left\{v_{k-1}, u_{k-1}\right\}$ and $N\left(v_{k+1}\right) \cap N\left(u_{k+1}\right)=\left\{v_{k+2}, u_{k+2}\right\}$.

\section{Main Results}

2.1 Theorem: $P_{n}^{2}$ is not a prime cordial graph for $n=3,4,5,7$.

Proof: If $v_{1}, v_{2}, \ldots, v_{n}$ are the vertices of path $P_{n}$ then $P_{n}^{2}$ is the graph with $\left|V\left(P_{n}^{2}\right)\right|=n$ and $\left|E\left(P_{n}^{2}\right)\right|=2 n-3$.

For the graph $P_{3}^{2}$ the possible pairs of labels of adjacent vertices are $(1,2),(1,3),(2,3)$. Then obviously $e_{f}(0)=0, e_{f}(1)=$ 3. Thus, $\left|e_{f}(0)-e_{f}(1)\right|=3$ and in all the possible assignments of vertex labels we have $\left|e_{f}(0)-e_{f}(1)\right|>1$. Therefore $P_{3}^{2}$ is not a prime cordial graph.

For the graph $P_{4}^{2}$ the possible pairs of labels of adjacent vertices are $(1,2),(1,3),(1,4),(2,3),(2,4),(3,4)$. Then obviously $e_{f}(0)=1, e_{f}(1)=5$. That is, $\left|e_{f}(0)-e_{f}(1)\right|=4$ and in all the possible assignments we have $\left|e_{f}(0)-e_{f}(1)\right|>1$. Therefore $P_{4}^{2}$ is not a prime cordial graph.

For the graph $P_{5}^{2}$ the possible pairs of labels of adjacent vertices are $(1,2),(1,3),(1,4),(1,5),(2,3),(2,4),(2,5),(3,4),(3,5)$, $(4,5)$. Then obviously $e_{f}(0)=1, e_{f}(1)=9$. That is, $\left|e_{f}(0)-e_{f}(1)\right|=8$ and in all the possible assignments we have $\left|e_{f}(0)-e_{f}(1)\right|>1$. Therefore $P_{5}^{2}$ is not a prime cordial graph.

Now for the graph $P_{7}^{2}$ the possible pairs of labels of adjacent vertices are $(1,2),(1,3),(1,4),(1,5),(1,6),(1,7),(2,3)$, $(2,4),(2,5),(2,6),(2,7),(3,4),(3,5),(3,6),(3,7),(4,5),(4,6),(4,7),(5,6),(5,7),(6,7)$. Then obviously $e_{f}(0)=4, e_{f}(1)=$ 17. That is, $\left|e_{f}(0)-e_{f}(1)\right|=13$ and in all the possible assignments we have $\left|e_{f}(0)-e_{f}(1)\right|>1$. Therefore $P_{7}^{2}$ is not a prime cordial graph.

Hence $P_{n}^{2}$ is not a prime cordial graph for $n=3,4,5,7$.

2.2 Theorem: $P_{n}^{2}$ is a prime cordial graph for $n=6$ and $n \geq 8$.

Proof: Let $v_{1}, v_{2}, \ldots, v_{n}$ be the vertices of path $P_{n}$. To define $f: V\left(P_{n}^{2}\right) \rightarrow\{1,2,3, \ldots, n\}$ we consider following three cases.

Case 1: $n=6,8,9$

The graphs $P_{6}^{2}, P_{8}^{2}$ and $P_{9}^{2}$ are to be dealt separately and their prime cordial labeling are shown in Figure 1 .

Case $2: n$ is even, $n \geq 10$

$$
\begin{array}{ll}
f\left(v_{1}\right)=1, & \\
f\left(v_{i+1}\right)=2^{i} ; & 1 \leq i \leq 3 \\
f\left(v_{4+i}\right)=2 i+10 ; & 1 \leq i \leq \frac{n}{2}-5 \\
f\left(v_{\frac{n}{2}}\right)=6, & \\
f\left(v_{\frac{n}{2}+1}\right)=10, & \\
f\left(v_{\frac{n}{2}+2}\right)=3, & \\
f\left(v_{\frac{n}{2}+3}\right)=5, & \\
f\left(v_{\frac{n}{2}+3+i}\right)=2 i+5 ; & 1 \leq i \leq \frac{n}{2}-3
\end{array}
$$

In view of the labeling pattern defined above we have $e_{f}(0)=e_{f}(1)+1=n-1$. 
Case 3: $n$ is odd, $n \geq 11$

$$
\begin{array}{ll}
f\left(v_{1}\right)=1, & \\
f\left(v_{i+1}\right)=2^{i} ; & 1 \leq i \leq 3 \\
f\left(v_{4+i}\right)=2 i+10 ; & 1 \leq i \leq\left\lfloor\frac{n}{2}\right\rfloor-5 \\
f\left(v_{\left\lfloor\frac{n}{2}\right\rfloor}\right)=6, & \\
f\left(v_{\left\lfloor\frac{n}{2}\right\rfloor+1}\right)=10, & \\
f\left(v_{\left\lfloor\frac{n}{2}\right\rfloor+2}\right)=3, & \\
f\left(v_{\left\lfloor\frac{n}{2}\right\rfloor+3}\right)=5, & \\
f\left(v_{\left\lfloor\frac{n}{2}\right\rfloor+3+i}\right)=2 i+5 ; & 1 \leq i \leq\left\lfloor\frac{n}{2}\right\rfloor-2
\end{array}
$$

In view of the labeling pattern defined above we have $e_{f}(0)+1=e_{f}(1)=n-1$.

Thus in all the above cases we have $\left|e_{f}(0)-e_{f}(1)\right| \leq 1$.

Hence $P_{n}^{2}$ is a prime cordial graph for $n=6$ and $n \geq 8$.

2.3 Illustration: Prime cordial labeling of the graph $P_{12}^{2}$ is shown in Figure 2.

2.4 Theorem: $C_{n}^{2}$ is not a prime cordial graph for $n \leq 9$.

Proof: If $v_{1}, v_{2}, \ldots, v_{n}$ are the vertices of cycle $C_{n}$ then $C_{n}^{2}$ is the graph with $\left|V\left(C_{n}^{2}\right)\right|=n$ and $\left|E\left(C_{n}^{2}\right)\right|=2 n$.

The graph $C_{3}^{2}$ is $C_{3}$. In the graph $C_{3}$ possible labels of adjacent vertices are $(1,2),(1,3),(2,3)$. Then obviously $e_{f}(0)=$ $0, e_{f}(1)=3$. That is, $\left|e_{f}(0)-e_{f}(1)\right|=3$. Therefore $C_{3}^{2}$ is not a prime cordial graph.

For the graph $C_{4}^{2}$ the possible pairs of labels of adjacent vertices are $(1,2),(1,3),(1,4),(2,3),(2,4),(3,4)$. Then obviously $e_{f}(0)=1, e_{f}(1)=5$. That is, $\left|e_{f}(0)-e_{f}(1)\right|=4$. Therefore $C_{4}^{2}$ is not a prime cordial graph.

For the graph $C_{5}^{2}$ the possible pairs of labels of adjacent vertices are $(1,2),(1,3),(1,4),(1,5),(2,3),(2,4),(2,5),(3,4)$, $(3,5),(4,5)$. Then obviously $e_{f}(0)=1, e_{f}(1)=9$. That is, $\left|e_{f}(0)-e_{f}(1)\right|=8$. Therefore $C_{5}^{2}$ is not a prime cordial graph.

For the graph $C_{6}^{2}$ the possible pairs of labels of adjacent vertices are $(1,2),(1,3),(1,4),(1,5),(1,6),(2,3),(2,4),(2,5),(2,6)$, $(3,4),(3,5),(3,6),(4,5),(4,6),(5,6)$. Then obviously $e_{f}(0)=4, e_{f}(1)=11$. That is, $\left|e_{f}(0)-e_{f}(1)\right|=7$ and in all the possible assignments we have $\left|e_{f}(0)-e_{f}(1)\right|>1$. Therefore $C_{6}^{2}$ is not a prime cordial graph.

For the graph $C_{7}^{2}$ the possible pairs of labels of adjacent vertices are $(1,2),(1,3),(1,4),(1,5),(1,6),(1,7),(2,3),(2,4),(2,5)$, $(2,6),(2,7),(3,4),(3,5),(3,6),(3,7),(4,5),(4,6),(4,7),(5,6),(5,7),(6,7)$. Then obviously $e_{f}(0)=4, e_{f}(1)=17$. That is, $\left|e_{f}(0)-e_{f}(1)\right|=13$ and in all the possible assignments we have $\left|e_{f}(0)-e_{f}(1)\right|>1$. Therefore $C_{7}^{2}$ is not a prime cordial graph.

For the graph $C_{8}^{2}$ the possible pairs of labels of adjacent vertices are $(1,2),(1,3),(1,4),(1,5),(1,6),(1,7),(1,8),(2,3),(2,4)$, $(2,5),(2,6),(2,7),(2,8),(3,4),(3,5),(3,6),(3,7),(3,8),(4,5),(4,6),(4,7),(4,8),(5,6),(5,7),(5,8),(6,7),(6,8),(7,8)$.

Then obviously $e_{f}(0)=7$. In order to satisfy edge condition for prime cordial graph it is essential to label eight edges with 0 and eight edges with 1 out of sixteen edges. But in all the possible assignments we are getting at most seven edge labels with 0 and at least nine edge labels with 1 . So $\left|e_{f}(0)-e_{f}(1)\right|>1$. Therefore $C_{8}^{2}$ is not a prime cordial graph.

Now for the graph $C_{9}^{2}$ in order to satisfy edge condition for prime cordial graph it is essential to label nine edges with 0 and nine edges with 1 out of eighteen edges. But in all the possible assignments we are getting at most seven edge labels with 0 and at least eleven edge labels with 1 . So $\left|e_{f}(0)-e_{f}(1)\right|>1$. Therefore $C_{9}^{2}$ is not a prime cordial graph.

Hence $C_{n}^{2}$ is not a prime cordial graph for $n \leq 9$.

2.5 Theorem: $C_{n}^{2}$ is a prime cordial graph for $n \geq 10$.

Proof: Let $v_{1}, v_{2}, \ldots, v_{n}$ be the vertices of cycle $C_{n}$. Then $C_{n}^{2}$ is the graph with $\left|V\left(C_{n}^{2}\right)\right|=n$ and $\left|E\left(C_{n}^{2}\right)\right|=2 n$. To define $f: V\left(C_{n}^{2}\right) \rightarrow\{1,2,3, \ldots, n\}$, we consider following three cases.

Case 1: $n=10$

The graph $C_{10}^{2}$ is to be dealt separately and its prime cordial labeling is shown in Figure 3.

Case 2: $n$ is odd, $n \geq 11$

Sub Case $1: n \equiv 1(\bmod 3)$ or $n \equiv 2(\bmod 3)$ 


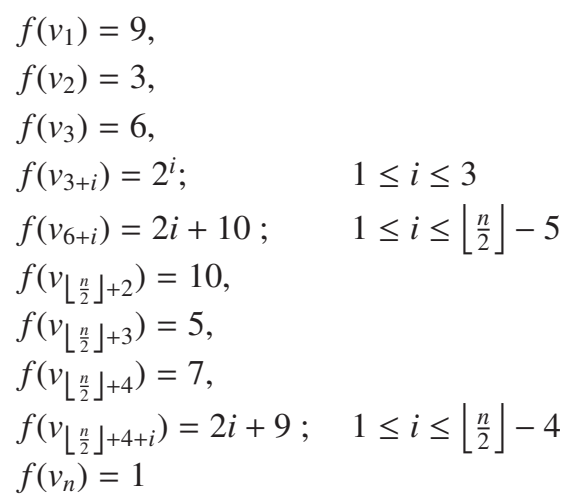

In view of the labeling pattern defined above, we have $e_{f}(0)=e_{f}(1)=n$.

Sub Case $2: n \equiv 0(\bmod 3)$

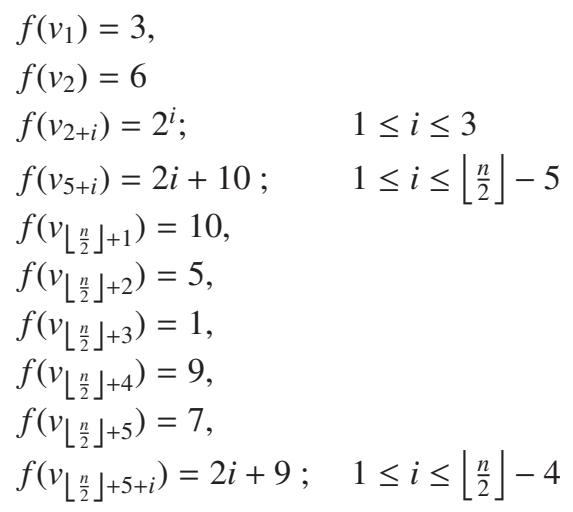

In view of the labeling pattern defined above, we have $e_{f}(0)=e_{f}(1)=n$.

Case 3: $n$ is even, $n \geq 12$

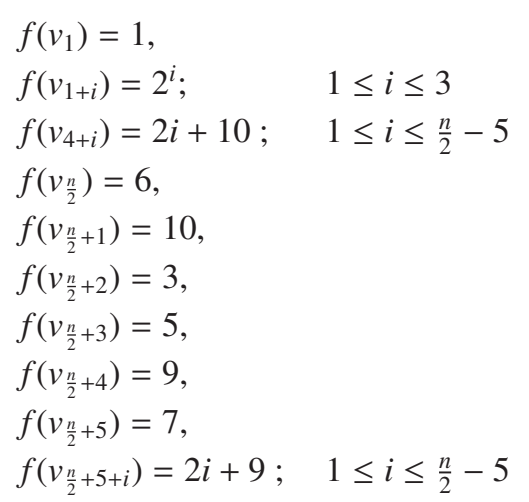

In view of the labeling pattern defined above, we have $e_{f}(0)=e_{f}(1)=n$.

Thus in the above cases, we have $\left|e_{f}(0)-e_{f}(1)\right| \leq 1$.

Hence $C_{n}^{2}$ is a prime cordial graph for $n \geq 10$.

2.6 Illustration: Prime cordial labeling of the graph $C_{15}^{2}$ is shown in Figure 4.

2.7 Theorem: $D_{2}\left(K_{1, n}\right)$ is a not a prime cordial graph for $n=2,3$.

Proof: Consider two copies of $K_{1, n}$. Let $v_{1}, v_{2}, \ldots, v_{n}$ be the pendant vertices of the first copy of $K_{1, n}$ and $v_{1}^{\prime}, v_{2}^{\prime}, \ldots, v_{n}^{\prime}$ be the pendant vertices of second copy of $K_{1, n}$ with $v$ and $v^{\prime}$ are the respective apex vertices. Let $G$ be the graph $D_{2}\left(K_{1, n}\right)$. Then $|V(G)|=2 n+2$ and $|E(G)|=4 n$.

In graph $D_{2}\left(K_{1,2}\right)$, in order to satisfy edge condition for prime cordial labeling it is essential to label four edges with 0 and four edges with 1 . But all the possible assignment of vertex labels give rise to 0 labels for at most three edges and 1 labels for at least five edges. Thus $\left|e_{f}(0)-e_{f}(1)\right|=2>1$. Hence $D_{2}\left(K_{1,2}\right)$ is not a prime cordial graph.

To satisfy edge condition for prime cordial labeling in the graph $D_{2}\left(K_{1,3}\right)$ it is essential to label six edges with 0 and six 
edges with 1. But all the possible assignments of vertex labels give rise to 0 labels for at most five edges and 1 labels for at least seven edges. Thus $\left|e_{f}(0)-e_{f}(1)\right|=2 \geq 1$. Hence $D_{2}\left(K_{1,3}\right)$ is not a prime cordial graph.

Hence $D_{2}\left(K_{1, n}\right)$ is a not a prime cordial graph for $n=2,3$.

2.8 Theorem: $D_{2}\left(K_{1, n}\right)$ is a prime cordial graph for $n \geq 4$.

Proof: Consider two copies of $K_{1, n}$. Let $v_{1}, v_{2}, \ldots, v_{n}$ be the pendant vertices of the first copy of $K_{1, n}$ and $v_{1}^{\prime}, v_{2}^{\prime}, \ldots, v_{n}^{\prime}$ be the pendant vertices of second copy of $K_{1, n}$ with $v$ and $v^{\prime}$ are the respective apex vertices. Let $G$ be the graph $D_{2}\left(K_{1, n}\right)$. Then $|V(G)|=2 n+2$ and $|E(G)|=4 n$. Choose a prime $p$ such that $2 p<2 n+2$ and $3 p \leq 2 n+2$ but $5 p>2 n+2$. We define $f: V(G) \rightarrow\{1,2,3, \ldots, 2 n+2\}$ as

$f(v)=2$,

$f\left(v_{1}\right)=1$,

$f\left(v^{\prime}\right)=2 p$,

$f\left(v_{1}^{\prime}\right)=p$,

$f\left(v_{2}^{\prime}\right)=3 p$

$f\left(v_{i}\right)=$ distinct even numbers between 4 to $2 n+2$ except $2 p, 2 \leq i \leq n$,

$f\left(v_{i}^{\prime}\right)=$ distinct odd numbers between 3 to $2 n+1$ except $p, 2 \leq i \leq n$.

In view of the labeling pattern defined above we have $e_{f}(0)=e_{f}(1)=2 n$. Thus we have $\left|e_{f}(0)-e_{f}(1)\right| \leq 1$.

Hence $D_{2}\left(K_{1, n}\right)$ is a prime cordial graph for $n \geq 4$.

2.9 Illustration: Prime cordial labeling of the graph $D_{2}\left(K_{1,5}\right)$ is shown in Figure 5.

2.10 Theorem: $D_{2}\left(B_{n, n}\right)$ is a prime cordial graph.

Proof: Consider two copies of $B_{n, n}$. Let $\left\{u, v, u_{i}, v_{i}, 1 \leq i \leq n\right\}$ and $\left\{u^{\prime}, v^{\prime}, u_{i}^{\prime}, v_{i}^{\prime}, 1 \leq i \leq n\right\}$ be the corresponding vertex sets of each copy of $B_{n, n}$. Let $G$ be the graph $D_{2}\left(B_{n, n}\right)$. Then $|V(G)|=4(n+1)$ and $|E(G)|=4(2 n+1)$. To define $f: V(G) \rightarrow\{1,2,3, \ldots, 4(n+1)\}$ we consider following two cases.

Case 1: $n=2$

The prime cordial labeling of $B_{2,2}$ is as shown in the Figure 6 .

Case 2: $n \geq 3$

Choose a prime $p$ such that $2 p<4(n+1)$ and $3 p \leq 4(n+1)$ but $5 p>4(n+1)$.

$f(u)=4$,

$f(v)=1$,

$f\left(u^{\prime}\right)=2$,

$f\left(v^{\prime}\right)=2 p$,

$f\left(v_{1}^{\prime}\right)=p$,

$f\left(v_{2}^{\prime}\right)=3 p$,

Define $f\left(u_{i}\right), 1 \leq i \leq n$ and $f\left(u_{i}^{\prime}\right), 1 \leq i \leq n-1$ distinct even numbers between 6 to $4(n+1)$ except $2 p$.

$f\left(u_{n}^{\prime}\right)=3$,

Define $f\left(v_{i}\right), 1 \leq i \leq n$ and $f\left(v_{i}^{\prime}\right), 3 \leq i \leq n$ distinct odd numbers between 5 to $4 n+3$ except $p$ and $3 p$.

In view of the labeling pattern defined above we have $e_{f}(0)=e_{f}(1)=2(2 n+1)$.

Thus in both the cases we have $\left|e_{f}(0)-e_{f}(1)\right| \leq 1$.

Hence $D_{2}\left(B_{n, n}\right)$ is a prime cordial graph.

2.11 Illustration: Prime cordial labeling of the graph $D_{2}\left(B_{5,5}\right)$ is shown in Figure 7.

2.12 Theorem: The graph obtained by mutual duplication of a pair of edges in $C_{n}$ is not a prime cordial graph for $n=3,4$.

Proof: Let $v_{1}, v_{2}, \ldots, v_{n}$ be the vertices of the first copy of cycle $C_{n}$ and $u_{1}, u_{2}, \ldots, u_{n}$ be the vertices of the second copy of cycle $C_{n}$. Denote by $G$ the graph obtained from mutual duplication of a pair of edges respectively from each of two copies of cycle $C_{n}$. Then $|V(G)|=2 n$ and $|E(G)|=2 n+4$.

In order to satisfy the edge condition for the graph obtained by mutual duplication of a pair of edges in $C_{3}$ it is essential to label five edges with 0 and five edges with 1 out of ten edges. But all the possible assignment of vertex labels give rise to label 0 for at most four edges and label 1 for at least six edges. Thus $\left|e_{f}(0)-e_{f}(1)\right|=2>1$. Hence the graph obtained by mutual duplication of a pair of edges in $C_{3}$ is not a prime cordial graph. 
In order to satisfy the edge condition for the graph obtained by mutual duplication of a pair of edges in $C_{4}$ it is essential to label six edges with 0 and six edges with 1 out of twelve edges. But all the possible assignment of vertex labels give rise to label 0 for at most five edges and label 1 for at least seven edges. Thus $\left|e_{f}(0)-e_{f}(1)\right|=2>1$. Hence the graph obtained by mutual duplication of a pair of edges in $C_{4}$ is not a prime cordial graph.

Hence the graph obtained by mutual duplication of a pair of edges in $C_{n}$ is not a prime cordial for $n=3,4$.

2.13 Theorem: The graph obtained by mutual duplication of a pair of edges in $C_{n}$ admits prime cordial labeling for $n \geq 5$.

Proof: Let $v_{1}, v_{2}, \ldots, v_{n}$ be the vertices of first copy of cycle $C_{n}$ and $u_{1}, u_{2}, \ldots, u_{n}$ be the vertices of the second copy of cycle $C_{n}$. Let $G$ be the graph obtained by mutual duplication of a pair of edges each respectively form each copy of cycle $C_{n}$. Then $|V(G)|=2 n$ and $|E(G)|=2 n+4$. To define $f: V(G) \rightarrow\{1,2,3, \ldots, 2 n+4\}$, we consider following two cases.

Case $1: n$ is odd, $n \geq 5$

Without loss of generality we may assume that the edge $e=v_{\frac{n+1}{2}} v_{\frac{n+3}{2}}$ from the first copy of cycle $C_{n}$ and the edge $e^{\prime}=u_{1} u_{2}$ from the second copy of cycle $C_{n}$ are mutually duplicated.

$$
\begin{array}{ll}
f\left(v_{i}\right)=n+6-2 i ; & 1 \leq i \leq \frac{n+5}{2} \\
f\left(v_{n+1-i}\right)=n+4+2 i ; & 1 \leq i \leq \frac{n-5}{2} \\
f\left(u_{1}\right)=2, & \\
f\left(u_{2}\right)=4, & \\
f\left(u_{3}\right)=10, & \\
f\left(u_{4}\right)=8, & \\
f\left(u_{n}\right)=6, & \\
f\left(u_{4+i}\right)=10+2 i ; & 1 \leq i \leq n-5
\end{array}
$$

Case 2: $n$ is even, $n \geq 6$

Without loss of generality we may assume that the edge $e=v_{\frac{n}{2}+1} v_{\frac{n}{2}+2}$ from the first copy of cycle $C_{n}$ and the edge $e^{\prime}=u_{1} u_{2}$ from the second copy of cycle $C_{n}$ are mutually duplicated.

$$
\begin{array}{ll}
f\left(v_{i}\right)=n+7-2 i ; & 1 \leq i \leq \frac{n}{2}+3 \\
f\left(v_{n+1-i}\right)=n+5+2 i ; & 1 \leq i \leq \frac{n}{2}-3 \\
f\left(u_{1}\right)=2, & \\
f\left(u_{2}\right)=4, & \\
f\left(u_{3}\right)=10, & \\
f\left(u_{4}\right)=8, & \\
f\left(u_{n}\right)=6, & \\
f\left(u_{4+i}\right)=10+2 i ; \quad 1 \leq i \leq n-5
\end{array}
$$

In view of the labeling pattern defined above we have $e_{f}(0)=e_{f}(1)=2 n+2$.

Thus in both the cases we have $\left|e_{f}(0)-e_{f}(1)\right| \leq 1$.

Hence the graph obtained by mutual duplication of a pair of edges in $C_{n}$ admits prime cordial labeling for $n \geq 5$.

2.14 Illustration: Prime cordial labeling of the graph obtained by mutual duplication of a pair of edges in $C_{9}$ is shown in Figure 8.

2.15 Theorem: The graph obtained by mutual duplication of a pair of vertices in $C_{n}$ is not a prime cordial graph for $n=3,4$.

Proof: Let $v_{1}, v_{2}, \ldots, v_{n}$ be the vertices of the first copy of cycle $C_{n}$ and let $u_{1}, u_{2}, \ldots, u_{n}$ be the vertices of the second copy of cycle $C_{n}$. Let $G$ be the graph obtained by mutual duplication of a pair of vertices each respectively from each copy of cycle $C_{n}$. Then $|V(G)|=2 n$ and $|E(G)|=2 n+4$.

In order to satisfy the edge condition for the graph obtained by mutual duplication of a pair of vertices in $C_{3}$ it is essential to label five edges with 0 and five edges with 1 out of ten edges. But all the possible assignment of vertex labels give rise to label 0 for at most four edges and label 1 for at least six edges. Thus $\left|e_{f}(0)-e_{f}(1)\right|=2>1$. Hence the graph obtained by mutual duplication of a pair of vertices in $C_{3}$ is not a prime cordial graph.

In order to satisfy the edge condition for the graph obtained by mutual duplication of a pair of vertices in $C_{4}$ it is essential to label six edges with 0 and six edges with 1 out of twelve edges. But all the possible assignment of vertex labels give 
rise to label 0 for at most five edges and label 1 for at least seven edges. Thus $\left|e_{f}(0)-e_{f}(1)\right|=2>1$. Hence the graph obtained by mutual duplication of a pair of vertices in $C_{4}$ is not a prime cordial graph.

Hence the graph obtained by mutual duplication of a pair of vertices in $C_{n}$ is not prime cordial for $n=3,4$.

2.16 Theorem: The graph obtained by mutual duplication of a pair of vertices in $C_{n}$ admits prime cordial labeling for $n \geq 5$.

Proof: Let $v_{1}, v_{2}, \ldots, v_{n}$ be the vertices of the first copy of cycle $C_{n}$ and $u_{1}, u_{2}, \ldots, u_{n}$ be the vertices of the second copy of cycle $C_{n}$. Let $G$ be the graph obtained by mutual duplication of a pair of vertices each respectively from each of two copies of $C_{n}$. Then $|V(G)|=2 n$ and $|E(G)|=2 n+4$. To define $f: V(G) \rightarrow\{1,2,3, \ldots, 2 n+4\}$ we consider following two cases.

Case 1: $n$ is odd, $n \geq 5$

Without loss of generality we may assume that the vertex $v_{\frac{n+3}{2}}$ from the first copy of cycle $C_{n}$ and the vertex $u_{1}$ from the second copy of cycle $C_{n}$ are mutually duplicated.

$$
\begin{array}{ll}
f\left(v_{i}\right)=n+2-2 i ; & 1 \leq i \leq \frac{n-3}{2} \\
f\left(v_{\frac{n+3}{2}-2}\right)=1, & \\
f\left(v_{\frac{n+3}{2}-1}\right)=2 n-4, & \\
f\left(v_{\frac{n+3}{2}}\right)=2 n, & \\
f\left(v_{\frac{n+3}{2}}+1\right)=2 n-2, & \\
f\left(v_{\frac{n+3}{2}}+1+i\right)=2 n+1-2 i ; & 1 \leq i \leq \frac{n-5}{2} \\
f\left(u_{1}\right)=2 n-6, & \\
f\left(u_{i+1}\right)=2 n-4-4 i ; & 1 \leq i \leq \frac{n-3}{2} \\
f\left(u_{\frac{n-1}{2}+i}\right)=1+2 i ; & 1 \leq i \leq 3 \\
f\left(u_{n+1-i}\right)=2 n-6-4 i ; & 1 \leq i \leq \frac{n-5}{2}
\end{array}
$$

Case 2: $n$ is even, $n \geq 6$

Without loss of generality we may assume that the vertex $v_{\frac{n+2}{2}}$ from the first copy of cycle $C_{n}$ and the vertex $u_{1}$ from the second copy of cycle $C_{n}$ are mutually duplicated.

$$
\begin{array}{ll}
f\left(v_{i}\right)=n+5-2 i ; & 1 \leq i \leq \frac{n-4}{2} \\
f\left(v_{\frac{n+2}{2}-2}\right)=1, & \\
f\left(v_{\frac{n+2}{2}-1}\right)=2 n-4, & \\
f\left(v_{\frac{n+2}{2}}\right)=2 n, & \\
f\left(v_{\frac{n+2}{2}}+1\right)=2 n-2 ; & \\
f\left(v_{\frac{n+2}{2}}+1+i\right)=2 n+1-2 i ; & 1 \leq i \leq \frac{n-4}{2} \\
f\left(u_{1}\right)=2 n-6, & \\
f\left(u_{i+1}\right)=2 n-6-4 i ; & 1 \leq i \leq \frac{n-4}{2} \\
f\left(u_{\frac{n}{2}+i}\right)=3+2 i ; & 0 \leq i \leq 2 \\
f\left(u_{n+1-i}\right)=2 n-4-4 i ; & 1 \leq i \leq \frac{n-4}{2}
\end{array}
$$

In view of the labeling pattern defined above we have $e_{f}(0)=e_{f}(1)=2 n+2$.

Thus in both the cases we have $\left|e_{f}(0)-e_{f}(1)\right| \leq 1$.

Hence the graph obtained by mutual duplication of a pair of vertices in $C_{n}$ admits prime cordial labeling for $n \geq 5$.

2.17 Illustration: Prime cordial labeling of the graph obtained by mutual duplication of a pair of vertices in $C_{10}$ is shown in Figure 9.

\section{Concluding Remarks}

As every graph is not a prime cordial graph it is very interesting to investigate graph or graph families which admit prime cordial labeling. In this paper we have investigated some new results on prime cordial labeling. To investigate similar results for other graph families and in the context of different labeling problems is an open area of research.

\section{Acknowledgement}

The authors are highly thankful to anonymous referees for their suggestions and critical comments which led to substantial 
improvement in quality of investigations.

\section{References}

Cahit, I. (1987). Cordial Graphs: A weaker version of graceful and harmonious Graphs, Ars Combinatoria, 23, $201-207$.

Gallian, J. A. (2010). A dynamic survey of graph labeling, The Electronics Journal of Combinatorics, 17(\# DS6). [Online] Available: http://www.combinatorics.org/Surveys/ds6.pdf

Gross, J., Yellen, J. (1999). Graph Theory and its applications, CRC Press.

Sundaram, M., Ponraj, R. and Somasundram, S. (2005). Prime Cordial Labeling of graphs, J. Indian Acad. Math., 27(2), 373-390.

Vaidya, S.K. and Vihol, P.L. (2010). Prime cordial labeling for some graphs, Modern Applied Science, 4(8), 119-126. Available: http://www.ccsenet.org/journal/index.php/mas/article/download/6421/5520

Vaidya, S.K. and Vihol, P.L. (2011). Prime cordial labeling for some cycle related graphs, Int. J. of Open Problems in Computer Science and Mathematics, 3(5), 2010, p. 223-232. Available: http://www.ijopcm.org/
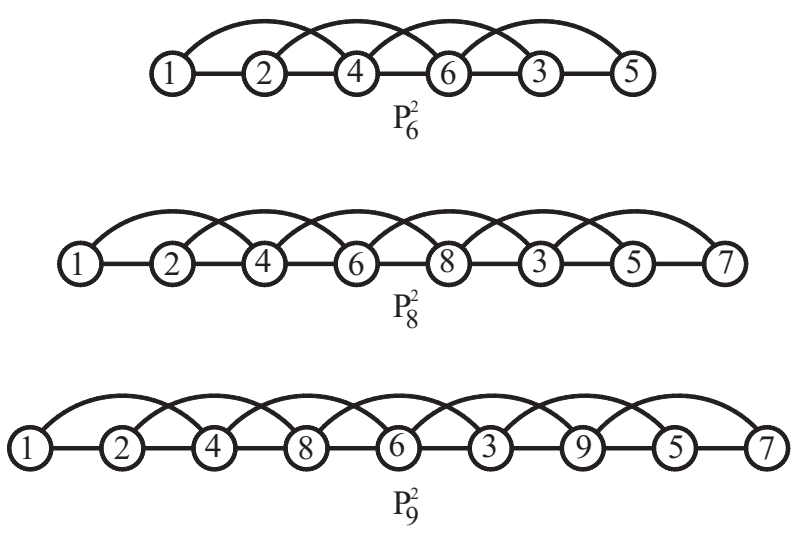

Figure 1. $P_{6}^{2}, P_{8}^{2}, P_{9}^{2}$ and their prime cordial labeling

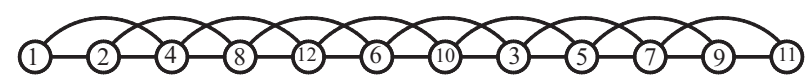

Figure 2. $P_{12}^{2}$ and its prime cordial labeling

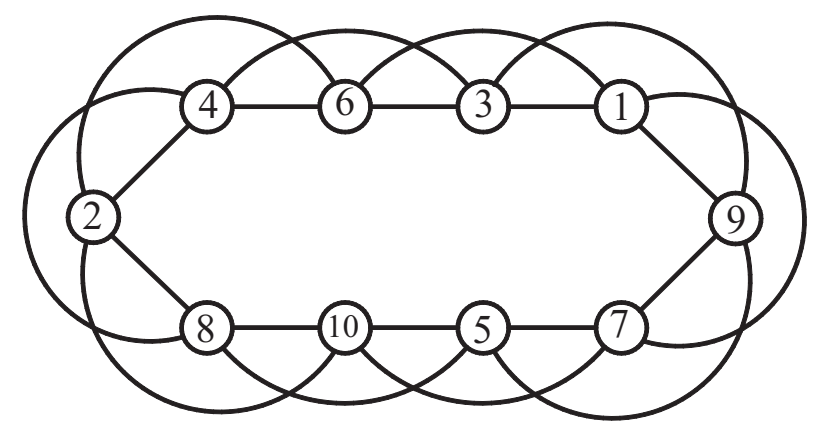

Figure 3. $C_{10}^{2}$ and its prime cordial labeling

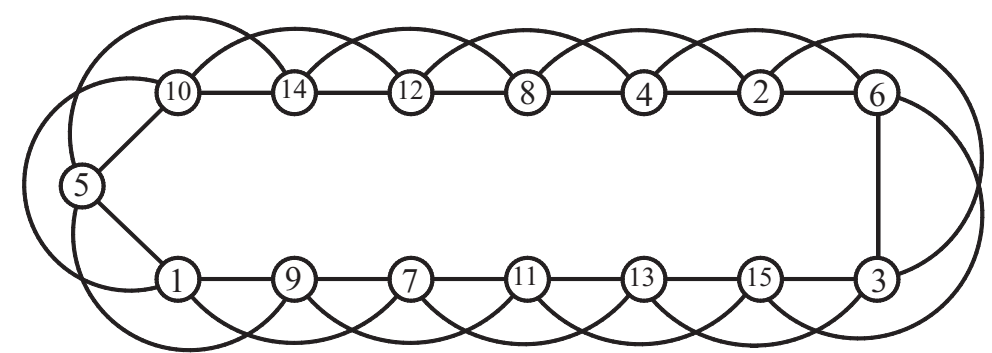

Figure 4. $C_{15}^{2}$ and its prime cordial labeling 


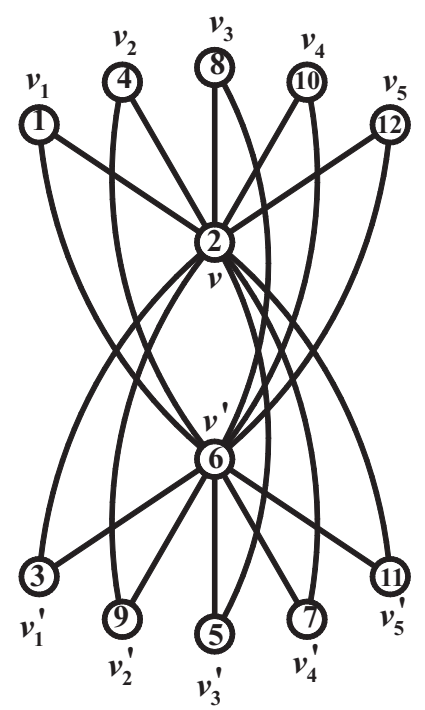

Figure 5. $D_{2}\left(K_{1,5}\right)$ and its prime cordial labeling

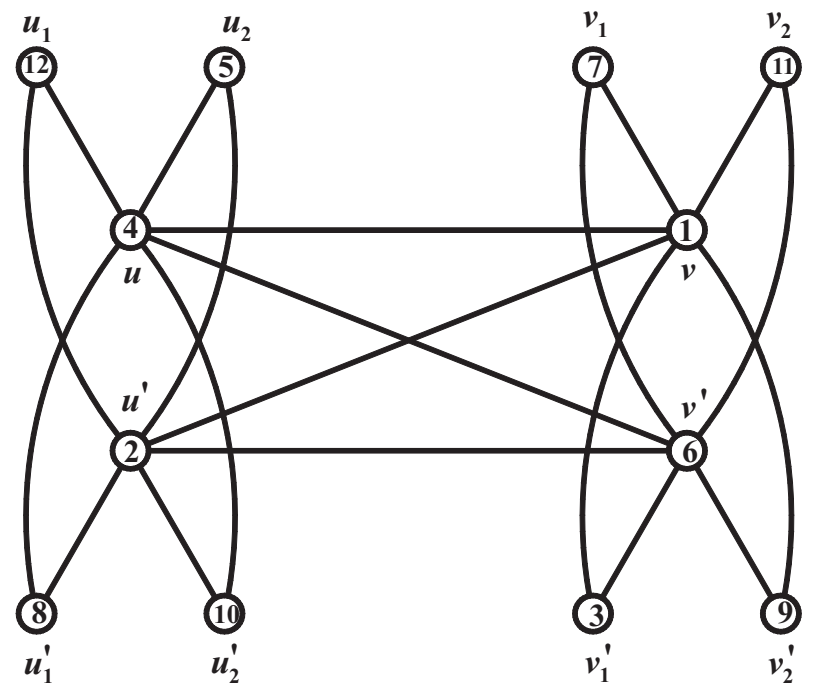

Figure 6. $D_{2}\left(B_{2,2}\right)$ and its prime cordial labeling

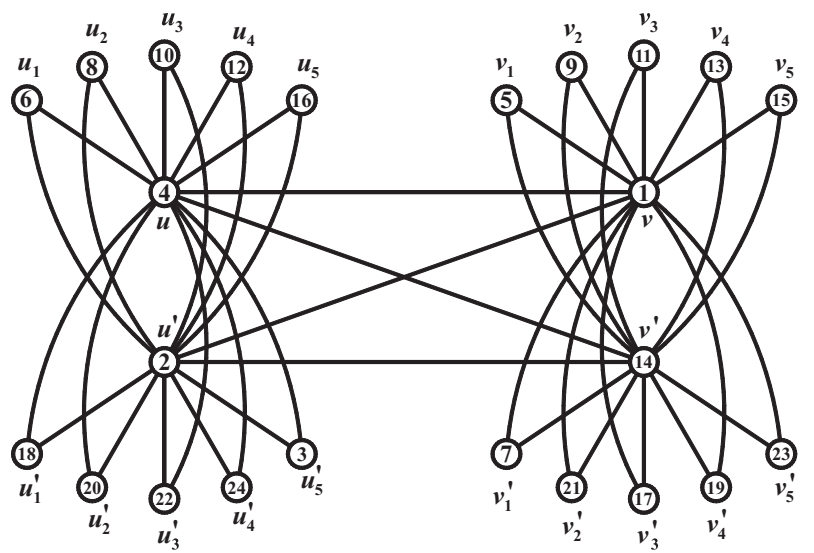

Figure 7. $D_{2}\left(B_{5,5}\right)$ and its prime cordial labeling 


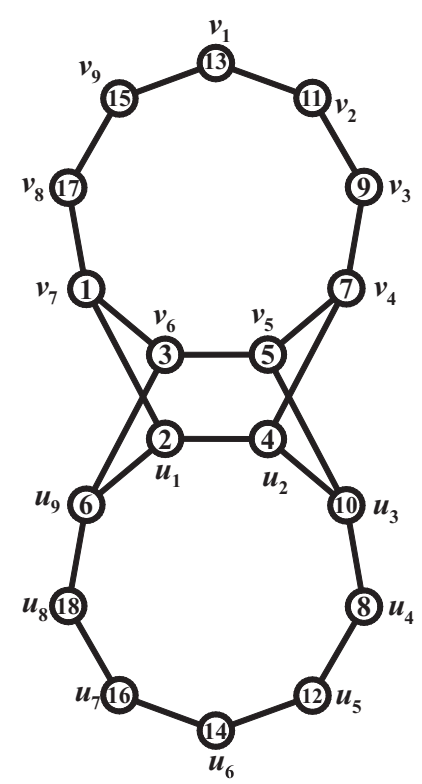

Figure 8 . The graph obtained by mutual duplication of a pair of edges in $C_{9}$ and its prime cordial labeling

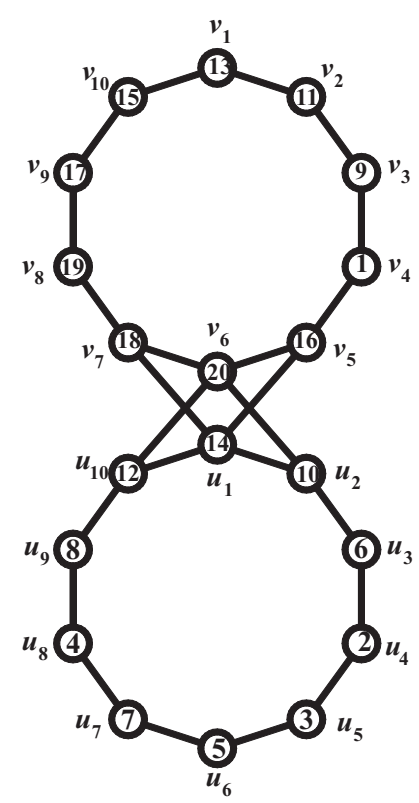

Figure 9 . The graph obtained by mutual duplication of a pair of vertices in $C_{10}$ and its prime cordial labeling 